\title{
APPLICATIONS OF EPIDEMIOLOGICAL TECHNIQUES
}

\author{
A. RALPH ROSENTHAL and JOHN R. THOMPSON \\ Leicester
}

\begin{abstract}
SUMMARY
Epidemiology is the study of disease occurrence in human populations. Its methodology could be used in health services research and medical audit. In fact, most ophthalmologists today should be using some form of epidemiological technique to evaluate their own practice and the service they are providing for their patients.
\end{abstract}

Epidemiology is the scientific study of disease occurrence in human populations. ${ }^{1}$ It seeks to describe patterns of disease and to discover causes; in both cases with an emphasis on quantification. Until recently the results of epidemiological studies were used mainly by Public Health workers interested in population-level interventions and by other medical researchers who, in their different ways, were also concerned with understanding the causal pathways that lead to disease. Increasingly, however, epidemiological findings are being used to allocate health care effort and resources. This new area of health services research has recently gained in importance in the United Kingdom with the advent of National Health Service reforms, in particular in the area of resource management and in the introduction of audit.

In designed experiments the researchers intervene to allocate a treatment or exposure, usually randomly, but opportunities for the use of experiments on human populations are limited by ethical and practical considerations. As a consequence epidemiologists, who usually rely on observational data, have had to be particularly inventive in the ways in which they design their studies. They often make use of existing national or regional data obtained from the reporting of notifiable diseases, disease registries or from death certification. Such information is limited and for more detailed work it

Correspondence to: A. Ralph Rosenthal, Department of Ophthalmology, Clinical Sciences Building, Leicester Royal Infirmary, PO Box 65, Leicester LE2 7LX, UK. becomes necessary to collect data on individuals through specially organised studies. Part of the art of epidemiology is to find a design that maintains scientific validity while staying within the constraints imposed by dealing with human populations.

In ophthalmology much of the most interesting epidemiological data has come from populationbased cross-sectional surveys such as Framingham and Beaver Dam in the United States. Currently there is considerable worldwide interest in extending this approach so that the same populations are resurveyed at regular intervals to observe disease progression.

\section{METHODS OF EPIDEMIOLOGICAL INVESTIGATION}

When we cannot use an experimental approach we must rely on observational data, either existing or specially collected. Observational studies are sometimes categorised as descriptive or analytic (aetiological), but such a distinction is not always helpful as a single study may have both descriptive and analytical elements. Descriptive work measures disease frequency within particular groups of people. This may involve incidence, the proportion of people who develop the disease in a given period of time; prevalence, the proportion of people who have the disease at any one time; or the consequences of disease such as survival times or mortality rates.

Existing data sources are often used in descriptive studies and they may well be detailed enough to enable the measures of disease frequency to be analysed by place, time or by basic demographic variables such as age, sex, race or social class. In this way we may obtain the first clues to the cause of a disease which may lead to further investigations. Thus, why is cataract so common in British Asians? Are there clusters of children born with anophthalmia? Why is the proportion of blind registration due to age-related macular degeneration increasing? 
Descriptive studies can also alert the medical community to what types of patients are most likely to be affected by the disease, and help in the planning for health and medical care facilities locally, regionally and nationally.

An analytic study tries to explain the observed patterns of the occurrence of a disease by identifying causal or aetiological factors. The two most commonly used analytic designs are the case-control study and the cohort study, which both seek to discover whether a disease outcome is associated with an earlier exposure. Until recently epidemiologists would talk of these designs as being opposite approaches, because a cohort study starts with exposed and unexposed people and follows them forward in time to observe disease outcome while a case-control study starts with diseased and diseasefree groups and works back in time to trace exposure. This is not a helpful distinction and has been called the 'trohoc fallacy'. In actuality both designs seek to study a population through time and to ask the same questions as to causality. They differ in the way that they sample subjects, the case-control design being more efficient when the disease is rare. Understanding the similarities between these two designs has led to the development of hybrid designs such as when cases and controls are selected from within the subjects recruited for a cohort study. The objective of this design is increased efficiency.

\section{HOSPITAL-BASED DESCRIPTIVE STUDIES}

An example of a major descriptive study which was undertaken in Leicester is the organisation, between 1980 and 1985, of an ophthalmic outpatient index. All patients seen in the Eye Department at the Leicester Royal Infirmary (the only Eye Department for the entire county) were studied. The Eye Casualty Department, ${ }^{2}$ the general and special clinics and the orthoptic and paediatric clinics were all included in the study. From this index the demand incidence of various ophthalmic disorders, the morbidity rates relating to specific ophthalmic entities and an overall view of eye disease in a county of nearly a million people was obtained.

One can do health services research in a similar way. For example, we could study a definite clinical service over a certain period to look for changes and then attempt to ascertain why the changes have occurred. We have just completed a study of this type looking at our Eye Casualty Department, which was studied originally in 1981-2. In 1992-3 there was a sixfold increase in the number of Eye Casualty patients coming to the Leicester Royal Infirmary compared with 1981-2. However, there has only been a $3 \%$ increase in the population of Leicestershire. A further study has therefore been initiated to find out the reasons for this increase in demand.

\section{POPULATION-BASED STUDIES}

We have carried out several population-based crosssectional surveys in Melton Mowbray, a town in the northeast of Leicestershire which has a population of about 32000 people. It is perfectly suited for population-based studies because it has a single group general practice that runs a computerised agesex registry which is regularly updated by noting patients new to the practice, deaths and patients who move out of the area. This sampling frame has allowed us to select patients randomly. Our first study looked at the prevalence of the chronic ophthalmological diseases in the over-75 year age group. ${ }^{3}$ In particular, we collected data relating to age-related macular degeneration, chronic simple glaucoma and cataract. We also studied the refractive error and near and distance visual acuity of the sample, enabling us to investigate the prevalence of blindness and partial sight and to relate it to registration. We have since used continuous national monitoring of death certificates for everyone in the original cohort to investigate mortality rates in relation to visual impairment, ${ }^{4}$ and very recently a second eye survey of age-related macular degeneration and age-related cataract has started. In the new study we are planning repeated examinations of the same subjects in order to observe the natural history of eye disease and to relate changes to suspected risk factors.

In other Melton studies we have looked at the prevalence of diabetic retinopathy in people with insulin-dependent and non-insulin-dependent diabetes. 5 Working closely with the general practitioners we identified everyone with diabetes in their practice and hence were able to study the ophthalmic consequences of diabetes in a representative English community.

\section{OTHER OBSERVATIONAL RESEARCH DESIGNS}

The greatest disadvantage of cross-sectional studies is that they may rely on memory to establish both the timing of events and earlier values of suspected risk factors. The ideal study design would follow diseasefree subjects by continuous surveillance to observe just when disease occurs. This is rarely possible unless the disease is such that it leads people to seek medical help as soon as it develops - a situation that does not apply to most eye diseases, which tend to develop slowly. The new Melton Eye Survey, with its planned resurveying of a random selection of subjects, is an attempt to go some way towards that ideal design. An alternative, suitable in other circumstances, would be to follow only those people identified in the cross-sectional study as having early disease.

Numerous case-control studies have been initiated 
to look at risk factors for eye disease. They have generally produced inconclusive results. The reasons are partly that they have attempted to measure too many factors; partly that the factors that appear to be important, such as diet and sunlight exposure, are hard to measure and are even harder to relate back in time; and partly that none of the relative risks is particularly large, making the studies very susceptible to bias.

Disease registries, though difficult to maintain, can be very useful. Where successfully organised they give a changing picture of the pattern of disease and can act as the source of subjects for analytic studies. In Leicester, we have used a registry to record and then study all cases of uveitis seen in the Uveitis Clinic at the Leicester Royal Infirmary. This registry provides a broad-based view of the patterns of uveitis that occur naturally within Leicestershire.

Britain has a well-established Blind Registry which has been used to provide important information relating to visual handicap. It can be used to study both visual disability and the community response to it. Although known to be incomplete, it is our opinion that this registry has been somewhat maligned and underused over the years. It is a unique tool which could provide important data relating to blindness and visual handicap.

\section{MEDICAL AUDIT}

With the recent emphasis within the National Health Service on medical audit as a discipline, we think it is important to look at medical audit as an epidemiological tool. In medical audit one evaluates what is currently operational to see whether standards, which have already been set, are being maintained. This type of investigation often comes close to descriptive epidemiology and epidemiologists should be aware of the opportunities for making use of data collected for audit.

Conversely audit can gain from epidemiology. Medical staff within the National Health Service are expected to be involved in medical audit. It is therefore imperative to make use of epidemiological research in setting standards and to understand that simple epidemiological techniques can be applied to medical audit studies.

\section{REFERENCES}

1. Friedman GD. Primer of epidemiology. New York: McGraw-Hill, 1974.

2. Chiapella A, Rosenthal AR. One year in an eye casualty clinic. Br J Ophthalmol 1985;69:865-70.

3. Gibson JM, Rosenthal AR, Lavery J. A study of the prevalence of eye disease in the elderly in an English community. Trans Ophthalmol Soc UK 1985;104: 196-203.

4. Thompson JR, Sparrow JM, Gibson JM, Rosenthal AR. Cataract and survival in an elderly non-diabetic population. Arch Ophthalmol 1993;111:675-9.

5. McLeod BK, Thompson JR, Rosenthal AR. The prevalence of retinopathy in insulin-requiring diabetic patients in an English country town. Eye 1988; 2:424-30.

6. Sparrow JM, McLeod BK, Smith TDW, Birch MK, Rosenthal AR. The prevalence of diabetic retinopathy and maculopathy and their risk factors in the noninsulin-treated patients of an English town. Eye 1993;7:158-63.

7. Thompson JR, Li D, Rosenthal AR. Recent trends in the registration of blindness and partial sight in Leicestershire. Br J Ophthalmol 1988;73:95-9. 